\title{
Title: Fantastic Plastic? Experimental evaluation of polyurethane bone substitutes as proxies for human bone in trauma simulations
}

\author{
1.0 Introduction \\ In cases involving the analysis of skeletal remains biological anthropologists can often \\ provide unique contributions to assist the pathologist and law enforcement agencies. One \\ such area is in the recognition of injuries to bone where investigations of past conflict \\ through analysis of archaeological burials have brought particular focus to injuries caused \\ through violence. Such work generally relies on comparisons with documented injuries. \\ In the case of more obscure or unusual mechanisms of injury, or when looking at past \\ populations a known comparator may not be available, for example in the case of archaic \\ weapons that are no longer in use. The best option to resolve uncertainty in such cases is \\ through actualistic experiments.
}

Experimentation in controlled circumstances is attractive, but raises questions regarding suitable test samples. In simulating skeletal trauma human cadaveric samples will obviously produce results that are very close to those that would be expected in a living individual but are not necessarily the most desirable option. Human cadavers are often difficult to obtain, carry infection risks and have issues of variability between samples. Aside from these points using human remains for such work is fraught with ethical concerns and often legal complications that rule them out for many researchers. Bone from non-human animals is a promising alternative, but is complicated in respect of anatomical differences between humans and other species. With regard to some parts of the skeleton such as ribs or flat bones such as the scapula, the overall form of some mammalian bones may be sufficiently similar to humans to make these a reasonable proxy. However, the unique size and form of the human cranium remains an intractable problem as non-human crania are so different in size, shape and thickness that results obtained from them are of limited value. This point has particular relevance to trauma as the head is a common target in assaults and is also the part of the skeleton where patterns of injury are most easily recognized.

A potential alternative is presented by the commercial availability of synthetic bone substitutes formed from polyurethane. These products offer several immediate advantages over human cadavers or non-human animal bone. They can be obtained quickly in variable quantities, each specimen is identical and they avoid complications of legality, ethics and infection. It would therefore seem that such material would be an obvious choice, however, this point hinges on the extent to which these replicas respond to dynamic impacts in ways that are analogous to real bone. In a series of papers Thali et al. $[1,2,3,4]$ claimed that similar replicas produced results that were highly accurate with regard to ballistic and blunt-force trauma, although these studies concentrated largely on simulated soft tissue at bullet entrance and exit points and the general appearance of fracture patterns at a gross scale. Thali et al. $[1,2,3,4]$ focused on a small range of modern firearms and a mechanism of blunt trauma, if such samples are viable substitutes for human crania they should hold equal potential for investigations of other mechanisms of injury. 
The current paper presents a pilot study designed to test this issue using a range of projectiles and mechanisms of launching them. Polyurethane bone substitute (PBS) samples were impacted with high, medium and low velocity projectiles shot using three different classes of modern and archaic weapons. The resultant defects in the samples were examined both grossly and in detail in order to assess the extent to which they resembled those produced in experimental animal bone samples and published examples of bony trauma in humans.

\subsection{Materials and Methods}

\subsection{Polyurethane Bone Substitute (PBS)}

The synthetic bone samples tested in the current study were obtained from Synbone AG (Malans, Switzerland) [5]. These products are marketed primarily as anatomical models for surgical training. The manufacturers do not give specific details about the composition of these products but describe them as being made of "specially formulated polyurethane foam" which have "in some instances the mechanical properties of natural bone" [5]. In addition to replicas of individual bones and particular portions of the skeleton for practicing surgical techniques, the suppliers also offer generic geometric forms for ballistic tests. These latter are supplied as flat plates, and hollow cylinders and spheres, representing flat bones, generalized long bones and crania respectively. The samples used in the current study were purchased by the first author's institution at the standard commercial rate.

\subsection{Testing low, medium and high velocity impacts}

The responses to impact of both flat plates and spheres of PBS were tested by firing shots at them using three different weapon types, modern rifles, a black powder musket and a crossbow. These weapons deliver differently constructed projectiles with stark differences in velocity and discharge energy (Table 1). The modern rifles were firstly a $7.62 \mathrm{~mm}$ Parker Hale T4 target rifle discharging a 7.62 x $51 \mathrm{~mm}$ NATO Full Metal Jacket (FMJ) bullet, a military round common internationally (Pretoria Metal Pressings, South Africa). The second was a Tikka model 550 rifle firing a .243" 100 grain Winchester Soft Point bullet, generally used for sport and hunting (Prvi Partizan, Serbia). The black powder musket was a replica 1861 model .58 calibre carbine discharging solid lead minié balls measuring (13.5mm diameter). The crossbow was a Jaguar $175 \mathrm{lb}$ recurve crossbow loaded with 17 1/4 inch long Perfectline alloy bolts fitted with conical target heads $(8.0 \mathrm{~mm}$ diameter).

\subsection{Experimental conditions and samples}

To comply with local police health and safety requirements the samples were shot in a prepared pit $1.5 \mathrm{~m}$ deep. The bottom $0.8 \mathrm{~m}$ of fill consisted of sieved sand to reduce risk of ricochet. The weapons were fired from a very close range of approximately $2 \mathrm{~m}$, secured in a fixed cradle to facilitate aiming and repeatability, at an angle of $65^{\circ}$ (Figure 1.). Shots were fired into flat PBS plates (5mm thick) and also PBS spheres of both $5 \mathrm{~mm}$ and $7 \mathrm{~mm}$ thickness. The latter are formed of two hemispheres held together with adhesive, with the consequent junction between the halves mimicking cranial sutures in a simplified form. The spheres also have an opening at the base which broadly simulates the openings in the cranium, particularly the foramen magnum. The work detailed in the 
current paper is part of a wider suite of ballistic experiments with relevance to Forensic Anthropology. One of these parallel projects [6] utilized cattle (Bos taurus) scapulae as proxies for human cranial bone in considering microscopic effects of gunshot trauma. Here fresh scapulae obtained from an abattoir from animals that had been slaughtered for food, were shot with minimal soft tissue $(<5 \mathrm{~mm}$ thick) adhering. The soft tissue was then removed using enzymatic detergent to allow examination of damage to the bone. These bovine samples also offered a useful comparison with aspects of the PBS samples in the current study as they were shot at the same time using the same weapons. Other studies have shown that areas of flat bone in large mammals respond in a uniform manner to ballistic trauma and so can be taken as a reasonable proxy for cranial and other flat bones in humans $[7,8]$-although they do not exhibit the kind of additional fractures that derive from the enclosed form of the cranial vault (see below).

\subsection{Simulating brain and associated soft tissue}

Initial tests were conducted firing the modern rifles into flat PBS plates and empty spheres to assess whether the different shapes would affect the way the material responded to impacts. In fact there were no gross differences between the two. Both exhibited simple ' internally bevelled' defects resembling those seen in gunshot injuries to areas of flat bone -i.e. where the internal/endocranial dimensions of the defect are larger than its external/ ectocranial dimensions $[9,10,11,12,13,14,15]$. However, the damage produced in the PBS spheres differed from gunshot wounds in real crania as there were no secondary or tertiary fractures of the kind frequently seen in cases of such trauma (see below). It was hypothesized that the lack of such fractures was related to the absence of soft tissue (brain, dura, etc.) inside these artificial 'crania'. Such soft tissues inside the cranium present a soft, semi fluid medium through which a hydraulic shockwave will form transmitting the kinetic energy of an impact to the cranial walls producing characteristic fractures [16]. In subsequent tests the spheres were therefore filled with ballistic gelatin (constituted at $10 \%$ by weight at $4^{\circ} \mathrm{C}$ ). This medium is widely used in ballistic tests as it approximates the density of human soft tissue $[17,18,19]$.

\subsection{Assessing outcomes}

The results were examined grossly to observe the size and morphology of entrance and exit wounds and the overall form of any additional (secondary and tertiary) fractures. These defects were then examined in greater detail using light microscopy in order to observe the extent to which any similarity to ballistic trauma in bone was consistent at a finer level. Lastly, the form of the bevelled entrance/exit defects produced by the firearm impacts was quantified mathematically by photographing them at $90^{\circ}$ and then tracing a digital path around the perimeter of the bevelled area using a vector drawing package (Adobe Illustrator). To facilitate comparison of overall form each photograph was then rotated and scaled to the same size on screen along the longest axis of the defect and radial lines were drawn from the centre to the outer margin of the beveled area at $10^{\circ}$ intervals. The length of each radial path was then measured using the same software in order to allow for statistical comparison. Other variables measured for comparison were the maximum diameter of the beveled area (in $\mathrm{mm}$ ) and the total area (measured in pixels) of beveling when standardized along the longest axis. 


\subsection{Results}

\subsection{Entrance/ exit beveling - gross observations}

The current project is considered a pilot study to assess the general potential of PBS as a proxy for human bone for use in larger studies. The work presented here focused on 12 PBS samples for each of the three projectile types used, with 12 cattle scapulae shot with the modern rifles included for comparison of beveled defects. The different weapon/ projectile types each produced distinctive patterns of damage in the PBS test samples that were easily distinguished from each other on gross examination. The initial tests involving flat plates and empty spheres shot with the modern rifles produced small rounded entrance/ exit defects where a cone shaped portion of the surface had been forced away from the rest of the sample to produce beveled margins as are frequently seen in ballistic trauma to bone (Figure 2). Such features are regarded as typical in penetrative injuries to areas of flat bone (most commonly the cranial vault, scapula, sternum or ilium) where the direction of bevelling is regarded as a standard feature for determining the direction in which a penetrative force has perforated bone $[9,10,11,12$, $13,14,19,20,21]$.

\subsection{Secondary-tertiary fractures}

A further distinguishing feature of cranial gunshot trauma is the presence of additional fractures which form, or travel, away from the point of impact. When bone fails in response to a high discharge of energy it behaves as a brittle material [22] and such fractures generally take the form either of linear fractures travelling outwards from the impact point (radiating or secondary fractures) or of concentric (or tertiary) fractures which form in a ring around the general area of impact (Figure 3). No secondary or tertiary fractures were produced in the flat plates and empty spheres. This conformed to expectations as such additional fractures are caused as an effect of releasing large amounts of kinetic injury into the soft tissues within the cranium. Such energy release is well documented as producing temporary cavitation effects through the action of a hydraulic shockwave which passes through the soft tissues adjacent to the primary wound track causing further damage through displacement and radial stretching [23, 24, 25]. In the enclosed space of the cranium this energy is transferred directly to the walls of the cranial vault causing it to fracture with the respective breaks originating at the impact point.

In spheres filled with ballistic gelatin the modern firearm impacts produced patterns of secondary and tertiary fractures generally consistent with those seen in cranial gunshot trauma (Figure 4). The black powder carbine produced similar patterns of fracturing although with concentric fractures that formed over a broader area around the impact point consistent with the larger calibre of the bullets. The projectiles used in this weapon are not only considerably greater in mass than modern bullets $(30 \mathrm{~g}$ as opposed to $<10 \mathrm{~g}$ ) but also being composed entirely of lead are more prone to deforming on entering the body, again contributing to both larger exit defects and greater tissue disruption than a modern bullet if it were fired at the same speed [26, 27]. An additional feature seen in the PBS spheres was the formation of keyhole shaped defects (Figure 4d) when bullets struck the spheres at a tangential angle. Again this is consistent with such impacts in real crania $[28,29]$. 


\section{3 (Gross) deviations from real bone}

The damage produced in PBS also displayed some features not seen in ballistic trauma to real crania. Most notably in the exit defects a pattern of fracturing was repeatedly produced that was characterized by 'stepped' fractures in which the radius of the defect varied by large intervals forming jagged corners around the margins. In this respect the damage in the PBS was quite unlike the generally regular and usually rounded/ ovoid defects produced by similar trauma to real bone. This effect was particularly pronounced for the samples shot with the black powder carbine, presumably due to the greater size of the projectile with its higher propensity to deform and impart energy throughout the cranium.

\subsection{Low velocity (crossbow) impacts}

The crossbow produced well defined, discrete entrance defects corresponding closely to the size and form of the projectiles. None of the crossbow impacts produced secondary or tertiary fractures at the entry points. The exit defects were all similarly comprised of a single point of failure from which a stellate pattern of linear fractures radiated out to a concentric fracture 30-40 mm from the centre (Figure $4 \mathrm{e}, \mathrm{f}$.). None of the respective impacts produced any fracturing that radiated beyond this initial concentric ring. The crossbow therefore produced a pattern of damage quite distinct from those caused by the firearms, consistent with the slower velocity of the projectiles and the lower level of energy involved (Table 1).

\subsection{Defect dimensions}

On close examination the defects produced in the PBS samples differed from those in both the experimental animal bone samples and from published examples of cranial gunshot trauma in several respects. Firstly, the size of the beveled areas differed between the PBS and real bone samples with generally larger defects seen in the PBS (Table 2). The two groups also differed in terms of the range of measurements and the variance within each sample. These differences were found to be significant using a T-test $(\mathrm{p}=0.004)$ although it should be noted that whilst the cattle bone values were normally distributed the PBS values were not and so the samples were also compared using a twosample Kolmogorov-Smirnov test which again found a significant difference $(\mathrm{p}=0.0008)$. The fact that the PBS values didn't conform to a normal distribution, whereas the bone samples did, further suggests that this material behaves differently to bone in response to impact.

\subsection{Defect shape}

Further differences were apparent in the shape of the beveled defects. The beveled areas in the bone samples were generally round or oval shaped with relatively gentle variations in diameter at different points around the circumference. The beveled areas in the PBS samples commonly had 'stepped' margins which varied in diameter repeatedly around the circumference. The boundaries between these angular steps were defined by thin radiating fractures not immediately obvious macroscopically. The defects in the PBS were consequently jagged and irregular in their overall form as opposed to being broadly sub-circular with generally smoother contours as seen in the real bone (Figure 5). These 
differences were also tested statistically using a two tailed T-test and a KolmogorovSmirnov two sample test (Table 2), the latter applied as the data were close to but not quite normally distributed. On comparing the radial measurements taken from the photographs there was a significant difference between the two groups (T-test: $\mathrm{p}=$ 0.0015 ; KS test: $p=0.004$ ). Further, it was suspected that the raw radial measurements might actually be masking greater quantitative differences in that whilst the means of the two samples were actually fairly close (42.44 and 44.12) the real source of variation lay in the 'stepped' margins seen in the PBS samples meaning that any individual measurement might considerably differ from its nearest neighbour. When the measurements were each subtracted from that of the next radial and the consequent values were re-tested, the result was even more significant (T test: $p=<0.0001$; KS test: $\mathrm{p}=0.002)$.

\subsection{Discussion}

\subsection{The bigger picture}

Whilst the simple bevelled defects produced by shooting both modern and obsolete forms of ammunition through flat PBS plates and empty spheres display general similarities to those seen in areas of flat bone, these lack the more complex patterns of breakage seen in real crania. However, the results of shooting PBS spheres filled with ballistic gelatin were more encouraging. On a gross level the latter are generally consistent with results obtained by Thali et al. $[1,2,3]$. The spheres shot with modern rifles in the current study compare favourably with published examples of modern cranial gunshot trauma [20, 21, $25,30,31,32,33,34]$. The black powder carbine produced patterns of damage consistent with archaeological examples interpreted as archaic firearms trauma including an example from the Netherlands dating from 1571 [35] two examples from Zürich from 1799 [36] and several recovered from the site of the Battle of the Little Bighorn, 1876 $[37,38]$.

\subsection{The devil in the detail}

The observed differences between the results in PBS and real bone ultimately derive from the differing microstructures of the two materials. Bone possesses complex mechanical properties resulting from its constituent materials and their arrangement in space [39]. All bone has a composite microstructure comprising crystals of the calcium compound hydroxyapatite $\left(\mathrm{Ca}_{10}\left(\mathrm{PO}_{4}\right)_{6}(\mathrm{OH})_{2}\right)$ set in an organic (protein) matrix of type 1 collagen fibrils $\left(\mathrm{C}_{2} \mathrm{H}_{5} \mathrm{NOC}_{5} \mathrm{H}_{9} \mathrm{NOC}_{5} \mathrm{H}_{10} \mathrm{NO}_{2}\right)$. Whilst the former conveys rigidity the latter confers a degree of flexibility and resistance to failure by making the bone less brittle. Cortical bone is formed in lamellar layers organized in a concentric, annular pattern in alternating directions around a central canal (Haversian systems). These Haversian structures run in a uniform local orientation making bone directionally 'grained'. The course taken by fractures is then influenced by this local orientation of bone fibres $[32,40]$. Further, the microstructure of bone has been shown to influence its response to ballistic trauma in complex ways at a microscopic level, including delamination, directional cortical bending and lateral deflection of portions of cortex. It has been demonstrated that such changes are recognizable to the extent that they can be used to differentiate between different types of ammunition microscopically [6]. In contrast to this organized complexity, polyurethane has an essentially uniform microstructure devoid of regular organization 
above the molecular level. These differences would explain the discrepancies noted in the response of the two materials to trauma both grossly and on close examination. With regard to these differences it is argued that polyurethane is not a useful proxy for studies investigating trauma to bone at anything below a very broad, superficial level. Further support is given to this point by Quenneville et al. [41] who found that stress and strain tests conducted on polyurethane long bone models demonstrated that these have tolerances that differ from real bone by significant amounts and therefore recommended that such are not used as proxies for human bone in experiments aimed at simulating road traffic collisions.

A further factor influencing the way fractures propagate in bone is the gross geometric form of individual bones. With regard to the skull, the various cranial bones exhibit complex shapes and variations in thickness throughout. Whilst some areas of the cranium can measure $<3 \mathrm{~mm}$ thickness [42], other areas are much thicker functioning as 'buttresses' (Figure 6) to strengthen particular areas [32]. These variations influence the course of fractures which will follow the line of least resistance and so deviate toward areas where the bone is thinner. Again, PBS differs in this regard in that it has a uniform thickness throughout.

\section{3 “We Prohibit that Murderous Art of Crossbowmen" (Pope Innocent II, AD1139)}

The crossbow was included to deliver impacts at lower velocity. Despite being a powerful example, the crossbow failed to induce secondary or tertiary fractures even though the bolts travelled through the full thickness of the gel-filled spheres to produce consequent 'exit wounds'. Again, this lack of complex fractures gives support to the idea that such patterns of damage are produced as the result of the shockwave effect produced by high velocity projectiles with sufficient energy to travel at hundreds of metres per second as opposed to the relatively slow velocities produced by the 'spring' mechanism of a bow (Table 1). This observation contradicts the supposition by Guiffra et al. [43] that crossbows would produce "extensive radiating fractures" because they are "high velocity weapons" (which they are not). Secondly, the area of 'cranium' punched outwards at the exit point of the bolt was sufficiently large that it might well not be recognized as a projectile wound if seen in a skeletonized individual. Such a defect might well be interpreted as blunt force trauma, rather than a projectile exit wound. As regards how to tell the difference (other than by noting the defect was opposite a small puncture on the opposite side of the cranium), this would involve attention to the direction of beveling at the wound margin. Bone is stronger in compression than in tension [39] and consequently when forces are sufficient to cause failure the resultant fractures will initiate from the side of the bone under tension (Figure 6 b., c.). In blunt force head injuries the forces acting on the cranium are delivered from the outside and so produce depressed defects that are beveled internally. Where concentric fractures are produced by forces from within the cranium -in this case the exiting crossbow bolt -the resultant defect (known as 'heaving fractures') will be externally beveled. These issues have been explored by Hart [16] in relation to gunshot wounds who supported this conclusion with forensic examples of such. Accordingly, such external beveling was present in the crossbow exit defects in the PBS spheres. Lastly, a salient point regarding the crossbow bolts used in the current study is that these had conical tips for target shooting (not dissimilar to bullets). This choice 
was made so that any differences in the results could be attributed to velocity/energy rather than variations in projectile form. It is intended to repeat this work to investigate the effects of different types of bolt head, as used variously in the Medieval period, such as triangular hunting heads or armour piercing heads.

\subsection{Conclusions}

Synthetic 'bones' of the kind tested in this study respond to ballistic impacts in ways that are superficially similar to the forms of damage that would be expected in human cranial bone. However, important differences were noted that render this material less useful for studies conducted at a more detailed level. In spite of this criticism a case remains for using simple synthetic models of this kind in ballistic experimentation. Despite the clear dissimilarities with the way real bone behaves at both a microscopic and macroscopic level, these spheres still exhibit patterns of damage that are a fair general approximation of those seen in bone. Whilst animal crania are too dissimilar to our own to be useful proxies and using human cadaveric material is ethically undesirable in many people's opinions (including our own) the latter is also problematic as discrepancies in the results of experiments simulating traumatic injury cannot easily be excluded from variation caused by differences in the strength and thickness of cranial bones both within and between individuals. In this regard synthetic bone substitutes actually possess a distinct advantage as they are uniform in size, shape and thickness and in this respect are arguably a better medium for simulating skeletal trauma than actual bone as any variations seen between samples must relate to differences in the mechanism of injury rather than confounding variables of differing thicknesses and irregular geometry in real crania. In the current study the damage patterns caused by the three different projectile/ weapon types used are clearly distinguishable from each other and as long as the above caveats are borne in mind such synthetic proxies offer a useful, relatively cheap and repeatable means of answering questions regarding the likely signatures of any other trauma mechanism researchers might wish to investigate.

\section{Acknowledgements}

Funding support for the current project was provided by Bournemouth University. We are indebted to Giles and Sandy Sturdy for kindly permitting the use of their land for the experiments described in this study and to Rob Sturdy for assisting with the preparations. We are grateful to the Assistant Chief Constable of Dorset Police for granting permission and to Sgt. John Hennessy (Firearms Training, Dorset Police, UK) for overseeing health and safety during the experiments. We also thank Dr. Luis Rios for kindly allowing us to use the photographs in Figure 3. Lastly thanks are due to Rosie Smith for assisting with data collection and recording. 


\section{Literature Cited}

[1] M.J. Thali, B.P. Kneubuehl, U. Zollinger, R. Dirnhofer, The "Skin -skull -brain model': a new instrument for the study of gunshot effects. Forensic Science International 125 (2002) 178-189.

[2] M.J. Thali, B.P. Kneubuehl, U. Zollinger, R. Dirnhofer, A study of the morphology of gunshot entrance wounds, in connection with their dynamic creation, utilizing the 'skinskull- brain model' Forensic Science International 125 (2002) 190-194.

[3] M.J. Thali, B.P. Kneubuehl, U. Zollinger, R. Dirnhofer, The dynamic development of the muzzle imprint by contact gunshot: high-speed documentation utilizing the 'skinskull-brain model'. Forensic Science International 127 (2002) 168-173.

[4] M.J. Thali, B.P. Kneubuehl, U. Zollinger, R. Dirnhofer, A "skin -skull -brain model" for the biomechanical reconstruction of blunt forces to the human head" Forensic Science International 125 (2002) 195-200.

[5] Synbone AG [Internet]. Malans, Switzerland: Statement Artificial vs. Human Bones [2007]. Available from:

https://www.synbone.ch/global/pdf/product_services/Statement_Artificial_vs_Human_B ones.pdf

[6] J. Rickman, M.J. Smith, Scanning Electron Microscope (SEM) analysis of gunshot defects to bone: an under-utilised source of information on ballistic trauma. Journal of Forensic Sciences (in press).

[7] Patten Report. Recommendations 69 and 70 Relating to Public Order Equipment : A Research Programme into Alternative Policing Approaches The Northern Ireland Office in association with the Association of Chief Police Officers, Belfast, 2000.

[8] M.J. Smith, M.B. Brickley, S.L. Leach, Experimental evidence for lithic projectile injuries: Improving recognition of an under-recognised phenomenon. Journal of Archaeological Science 34 (2007) 540-553.

[9] J. Bailey, P.D. Mitchell, A case for Sherlock Holmes: forensic investigation of a gunshot wound to the head dating from Victorian London. International Journal of Osteoarchaeology 17 (2007) 100-104.

[10] H.E. Berryman, S.A. Symes, Recognising gunshot and blunt cranial trauma through fracture interpretations, in: Forensic Osteology: Advances in the Identification of Human Remains, K.J. Reichs (Ed.). Charles Thomas, Illinois, 1998, pp. 333-352.

[11] P. Besant-Matthews, Examination and interpretation of rifled firearm injuries, in: The Pathology of Trauma, J.K. Mason, B.N. Purdue (Eds.) Arnold: London, 2000, pp.4774. 
[12] J.E. Buikstra, D.H. Ubelaker, Standards for Data Collection from Human Skeletal Remains. Arkansas Archaeological Survey: Arkansas, 1994.

[13] M. Nafte, Flesh and Bone-an Introduction to Forensic Anthropology. Carolina Academic Press: North Carolina, 2000.

[14] N.J. Sauer, The timing of injuries and manner of death: distinguishing among antemortem, perimortem and postmortem trauma, in: Forensic Osteology: Advances in the Identification of Human Remains, K.J. Reichs (Ed.) Charles Thomas, Illinois, 1998, pp. 321-332.

[15] O.C. Smith, E.J. Pope, S.A. Symes, Look until you see: Identification of trauma in skeletal material, in: Case Studies in Forensic Anthropology, D.W. Steadman (Ed.) Prentice Hall: New Jersey, 2003, pp. 138-154.

[16] G.O. Hart, Fracture pattern interpretation in the skull: differentiating blunt force from ballistics trauma using concentric fractures, Journal of Forensic Sciences 50 (2005) $1-6$.

[17] M.D. Alley, R. Schimizze, S.F. Son, Experimental modeling of explosive blastrelated traumatic brain injuries, NeuroImage 54 (2011) S45-S54.

[18] Y. Wen, C. Xu, H. Wang, A. Chen, R.C. Batra, Impact of steel spheres on ballistic gelatin at moderate velocities, International Journal of Impact Engineering 62 (2012) 142-151.

[19] M.V. Swain, D.C. Kieser, S. Shah, J.A. Kieser, Projectile penetration into ballistic gelatin, Journal of the Mechanical Behavior of Biomedical Materials 29 (2014) 385-392.

[20] G. Quatrehomme, M.Y. İşcan, Analysis of bevelling in gunshot entry wounds. Forensic Science International 93 (1998) 45-60.

[21] D.W. Steadman, W. Basler, M.J. Hochrein, D.F. Klein, J.C. Goodin, Domestic homicide investigations: an example from the United States, in: Handbook of Forensic Archaeology and Anthropology, S. Blau, D. Ubelaker (Eds.) Left Coast Press: Walnut Creek, 2011, pp. 351-362.

[22] D.C. Kieser, R. Riddell, J.A. Kieser, J.C. Theis, M.V. Swain, Bone Micro-Fracture Observations From Direct Impact of Slow Velocity Projectiles, Journal of Archives in Military Medicine 2 (2013) 1-6.

[23] G.W. Dufresne, Wound ballistics: recognizing wound potential. Part 1: characteristics of missiles and weapons, International Journal of Trauma Nursing 1 (1995) 4-10. 
[24] L.A. Farjo, T. Miclau, Ballistics and mechanisms of tissue wounding. Injury 28 (1997) C12-C27.

[25] M. Oehmichen, C. Meissner, H.G. König, H.B. Gehl, Gunshot injuries to the head and brain caused by low-velocity handguns and rifles. Forensic Science International 146 (2004) 111-120.

[26] P.J. Dougherty, H. Collins, Wound ballistics: mini ball versus full metal jacketed bullets -a comparison of Civil War and Spanish-American War firearms, Military Medicine 174 92009) 403-407.

[27] M. Garrat, Wound ballistics: exploring the wounding potential of penetrating projectile injury, Journal of Paramedic Practice 4 (2011) 201-207.

[28] K. Harada, R. Kuroda, M. Nakajima, A. Takizawa, K. Yoshida, An autopsy case of a decomposed body with keyhole gunshot wound and secondary skull fractures. Legal Medicine 14 (2012) 255-257.

[29] A.M. Jackson, J.G. Smirniotopoulos, L. Folio, Keyhole fracture of the skull. Military Medicine Radiology Corner 173 (2008) 1-4.

[30] B. Madea, M. Staak, Determination of the sequence of gunshot wounds in the skull, Journal of the Forensic Science Society 28 (1988) 321-328.

[31] G. Quatrehomme, M.Y. İşcan, Bevelling in exit gunshot wounds in bones, Forensic Science International 89 (1997) 93-101.

[32] T. Fenton, V.H. Stefan, L.A. Wood, N.J. Sauer, Symmetrical fracturing of the skull from midline contact gunshot wounds: reconstruction of individual death histories from skeletonized human remains. Journal of Forensic Sciences 50 (2005) 1-12.

[33] E. Susa, Forensic Anthropology in Hungary, in: Forensic Anthropology Case Studies from Europe, M.B. Brickley, R. Ferllini (Eds.) Charles Thomas, Springfield (Il), 2007.

[34] L. Ríos, A. García-Rubio, B. Martínez, L. Herrasti, F. Exteberria, Patterns of perimortem trauma in skeletons recovered from mass graves from the Spanish civil war (1936-9), in: C. Knüsel, M.J. Smith (Eds.) The Routledge Handbook of the Bioarchaeology of Human Conflict, Routledge, London, 2013, pp. 621-640.

[35] R. Schats, L.M. Kootker, R. Hermsen, G.R. Davies, M.L.P. Hoogland, The Alkmaar mass graves: a multidisciplinary approach to war victims and gunshot trauma in: $\mathrm{C}$. Knüsel, M.J. Smith (Eds.) The Routledge Handbook of the Bioarchaeology of Human Conflict, Routledge, London, 2013, pp. 455-472.

[36] C. Meyer, Osteological evidence for the battles of Zürich, 1799: a glimpse into soldiery of the past, International Journal of Osteoarchaeology 13 (2003) 252-257. 
[37] P. Willey, D.D. Scott, 'The bullets buzzed like bees': gunshot wounds in skeletons from the Battle of the Little Bighorn, International Journal of Osteoarchaeology 6 (1996) $15-27$.

[38] D.D. Scott, P. Willey, M.A. Connor, They Died with Custer: Soldiers' Bones from the Battle of the Little Bighorn, Norman, University of Oklahoma, 1998.

[39] P. Augat, S. Schorlemmer, The role of cortical bone and its microstructure in bone strength, Age and Ageing 35-S2 (2006) ii27-ii31.

[40] A. Benninghoff, Spaltlinien am knochen, eine methode zur ermittlung der architektur platter knochen, Verhandlungen Anatomische Gesellschaft 34 (1925) 189206.

[41] C.E. Quenneville, G.S. Greeley, C.E. Dunning, Evaluation of synthetic composite tibias for fracture testing using impact loads, Proceedings of the Institution of Mechanical Engineers, Part H: Journal of Engineering in Medicine 224 (2010) 1195 -1199.

[42] A.C. de Souza Fernandes, A.I. Trindade Neto, A.C. de Freitas, M. de Moraes, Dimensional analysis of the parietal bone in areas of surgical interest and relationship between parietal thickness and cephalic index, Journal of Oral and Maxillofacial Surgery 69 (2011) 2930-2935.

[43] V. Giuffra, A.L. Pejrani Baricco, B.M. Subbrizioc, G. Fornaciaria, Weapon-related cranial lesions from Medieval and Renaissance Turin, Italy. International Journal of Osteoarchaeology (2014) (online) DOI: 10.1002/oa.2334.

[44] P. Krenn, P. Kalaus, B. Hall, Material culture and military history: test-firing early modern small arms, Military History Review 42 (1995) 101-109. 


\section{Figure Captions}

1. Views of experimental arrangement and ammunition used. a. The adjustable cradle with Parker Hale rifle in place ready to fire through a chronograph into the test pit, b. 7.62 x $51 \mathrm{~mm}$ NATO Full Metal Jacket ammunition -longitudinal section reveals jacket over bullet tip, c. .243" Winchester jacketed soft-point longitudinal section reveals lead core visible at the projectile tip, d. Lead Minié balls -developed in the mid $19^{\text {th }}$ century with a conical profile and concave base designed to cause the bullet to expand and grip the barrel rifling to improve stability, e. Crossbow bolt tip with conical target point.

2. Views of bevelled defects produced in PBS samples by the modern hunting rifles. a. exit defect in empty sphere, b.'exit' defect in flat plate.

3. a. Illustration of radiating (secondary) and concentric (tertiary) fractures of the cranial vault in response to high energy impacts. b.-f. Cranial gunshot wounds in skeletal remains of individuals killed during the Spanish Civil War displaying 'classic' examples of gunshot entrance and exit defects and additional associated fractures. These injuries are likely to have been caused by M93 Mauser rifles which deliver a round at a weight and velocity in between those of the modern rifles and black powder musket used in the current study (photographs by kind permission of Luis Rios).

4. Patterns of damage produced in PBS spheres filled with ballistic gelatine. a., b. internal and external views of classic stellate and concentric fractures in an entrance defect produced by a 7.62 NATO round, c. entrance defect produced by black powder minié ball with stellate and concentric fractures, d. 'keyhole' defect produced by a tangential impact from a minié ball, e. discrete entrance defect produced by crossbow bolt lacking secondary/ tertiary fractures, f. reconstructed fragments at crossbow exit defect -stellate fracture centred on a single point of failure contained within a discrete secondary (concentric) fracture.

5. Comparison of the form of bevelled exit defects in PBS samples and real bone (human and animal) shot with modern firearms. The defects were photographed and the outlines traced to produce vector drawings. These were then standardised to the same dimensions along their longest axis and radial centroids were added at $10^{\circ}$ intervals to facilitate comparison of morphology (actual dimensions and area were compared separately) a. overlay of outlines of 8 defects in real bone, $b$. overlay of outlines of 8 defects in PBS samples, c., d. examples of photographic overlay in real bone and PBS, e. data values displayed as Gaussian curves to 
illustrate the extent of overlap/ separation between the two samples when comparing them in terms of different variables.

6. a. Frontal view of areas of facial buttressing influencing the course of fractures within the skull (after Fenton et al., 2005). b, c. schematic representation of the formation of concentric fractures surrounding significant impacts to the cranial vault, b. blunt force trauma -here all the mechanical force affecting the cranium is delivered externally producing a concentric fracture with an internally bevelled margin, c. high energy ballistic trauma -here the gases and kinetic energy discharged by the bullet produce counteracting forces from inside the cranium causing the associated concentric defect to bevel outwards (after Hart, 2005). $\mathrm{C}=$ bone stressed in compression, $\mathrm{T}=$ bone stressed under tension.

\section{Tables}

Table 1. Characteristics of the weapons and ammunition used in the experiments. Velocity data derived from: *Manufacturers website; ** Measured by present authors by chronograph; *** Averaged from published velocities for four black powder weapons of similar type and date [44].

\begin{tabular}{|c|c|c|c|c|c|c|}
\hline \multirow[b]{2}{*}{ Weapon } & \multicolumn{4}{|c|}{ Ammunition } & \multirow[b]{2}{*}{$\begin{array}{l}\text { Velocity } \\
\text { (m/s) }\end{array}$} & \multirow[b]{2}{*}{$\begin{array}{c}\text { Energy } \\
\text { (J) }\end{array}$} \\
\hline & Type & Calibre & $\begin{array}{l}\text { Weight } \\
\text { [grains] }\end{array}$ & $\begin{array}{l}\text { Weight } \\
\text { (grams) }\end{array}$ & & \\
\hline Tikka 550 & Winchester SP & .243" & 100 & 6.5 & $905^{\star}$ & 2660 \\
\hline Parker Hale T4 & Nato FMJ & 7.62 & 147 & 9.5 & $853^{\star \star}$ & 3456 \\
\hline 1861 Carbine & Minie Ball & 0.58 & 463 & 30.0 & $468^{\star \star \star}$ & 3285 \\
\hline Jaguar Crossbow & Perfectline alloy & 8.0 & 363 & 23.5 & $75^{*}$ & 66 \\
\hline
\end{tabular}

Table 2. Measurements of beveled areas produced by gunshots fired from the modern rifles. NN=Nearest Neighbour. *Images scaled to same size along their longest axis so measurements $=$ relative to overall form rather than absolute units.

\begin{tabular}{|l|r|r|r|r|c|}
\hline \multirow{2}{*}{} & \multicolumn{2}{|c|}{ Bone } & \multicolumn{2}{c|}{ PBS } & $\begin{array}{c}\text { Kolmogorov- } \\
\text { Smirnov Test }\end{array}$ \\
\cline { 2 - 6 } & \multicolumn{1}{|c|}{ Mean } & \multicolumn{1}{c|}{ St.Dev } & \multicolumn{1}{c|}{ Mean } & \multicolumn{1}{c|}{ St.Dev } & P-value \\
\hline Max. Diameter (mm) & 20.62 & 5.14 & 14.97 & 2.37 & 0.008 \\
\hline Radial Measurements $^{*}$ & 42.43 & 6.31 & 44.12 & 6.37 & 0.004 \\
\hline NN Differences* & 2.00 & 2.04 & 3.23 & 4.46 & 0.002 \\
\hline Comparative area* $^{*}$ & 11924.38 & 2304.02 & 12995.25 & 1997.81 & 0.023 \\
\hline
\end{tabular}

